\section{Modelagem hierárquica de determinantes associados a internações por condições sensíveis à atenção primária no Espírito Santo, Brasil}

\author{
Hierarchical modeling of determinants associated \\ with hospitalizations for ambulatory care sensitive \\ conditions in Espírito Santo State, Brazil
}

\author{
Modelización jerárquica de los determinantes \\ asociados a hospitalizaciones por ambulatory care \\ sensitive conditions en Espírito Santo, Brasil
}

\author{
1 Programa de Pós-graduação \\ em Saúde Coletiva, \\ Universidade Federal do \\ Espírito Santo, Vitória, Brasil. \\ 2 Escola Superior de Ciências, \\ Santa Casa de Misericórdia, \\ Vitória, Brasil. \\ Correspondência \\ R. G. Pazó \\ Programa de Pós-graduação \\ em Saúde Coletiva, \\ Universidade Federal do \\ Espirito Santo. \\ Av. Marechal Campos 1468, \\ Vitória, ES 29000-000, Brasil. \\ rosalvapazo@gmail.com
}

\begin{abstract}
The aim of this study was to investigate the relationship between health services organization and hospitalization rates for ambulatory care sensitive conditions after adjusting for socioeconomic and demographic variables in municipalities (counties) in Espírito Santo State, Brazil. In an ecological study, data were collected from the Brazilian Unified National Health System (SUS) on the following variables: hospitalization for ambulatory care sensitive conditions, city size, demographic and socioeconomic characteristics, and health services organization. Rates were analyzed by Poisson regression with robust variance. Models were adjusted for the total popula tion and age group. The explanatory variables were ordered hierarchically. Hospitalization rates for ambulatory care sensitive conditions were associated with illiteracy rate (RR: 1.08-1.17), proportion of beds in the SUS (RR: 1.09-1.12), urbanization (RR: 1.02-1.03), proportion of blacks (RR: 0.97-0.98), and health insurance coverage (RR: 0.97-0.98). Some determinants of hospitalization for ambulatory care sensitive conditions involve patterns of health services use and lie outside the scope of primary care.
\end{abstract}

Health Care Quality Indicators; Primary Health Care; Ecological Studies
Rosalva Grobério Pazó 1

Diana de Oliveira Frauches 2 Maria del Carmen Bisi Molina 1 Nágela Valadão Cade ${ }^{1}$

\section{Resumo}

O objetivo deste estudo foi investigar a associação entre a organização dos serviços de saúde e as taxas de internações hospitalares por condições sensiveis à atenção primária após ajuste para variáveis socioeconômicas e demográficas no contexto dos municípios do Espírito Santo, Brasil. Estudo ecológico das internações do Sistema Único de Saúde (SUS) com as variáveis: internações hospitalares por condições sensíveis à atenção primária, porte municipal, indicadores demográficos, socioeconômicos, organização e indicadores de desempenho dos serviços de saúde. Realizou-se análise multivariável por regressão de Poisson com ajuste robusto da variância. Foram ajustados modelos para a população total e por faixas etárias. Ordenaram-se as variáveis explicativas segundo modelo hierarquizado. Houve associação a risco de internações hospitalares por condições sensíveis à atenção primária com o percentual de analfabetismo (RR: 1,08-1,17), proporção de leitos SUS (RR: 1,09-1,12), urbanização (RR: 1,02-1,03), proporção de negros (RR: 0,97-0,98) e cobertura de plano de saúde (RR: 0,97-0,98). Há determinantes das internações hospitalares por condições sensíveis à atenção primária que implicam no padrão de utilização dos serviços e estão fora do escopo da atenção primária.

Indicadores de Qualidade em Assistência à Saúde; Atenção Primária à Saúde; Estudos Ecológicos 


\section{Introdução}

As internações por condições sensíveis à atenção primária (ICSAP) constituem um indicador de resultado utilizado para avaliar a acessibilidade e a eficácia dos cuidados primários de saúde, que pretende, a partir da atividade hospitalar, servir de medida da efetividade da atenção primária à saúde. Trata-se, portanto, de uma medida indireta para se avaliar a atenção primária à saúde 1,2 .

A justificativa para o indicador é que o atendimento ambulatorial oportuno e de qualidade a determinados problemas de saúde pode diminuir o risco de hospitalização 1,3. Os problemas de saúde que constituem as condições sensíveis à atenção primária, expressão derivada do inglês ambulatory care sensitive conditions (ACSC), são aqueles para os quais as ações próprias da atenção primária à saúde podem modificar a sua ocorrência ou evolução por meio da prevenção, do diagnóstico e do tratamento precoce. Isto é, o controle da enfermidade numa fase prévia a um estádio clínico que requeira a internação ou, no caso dos agravos crônicos, pelo controle, acompanhamento e redução das exacerbações ${ }^{3}$.

A lista brasileira de ICSAP, publicada em 2008, é composta por 19 grupos de diagnósticos: doenças preveníveis por imunização e por condições sensíveis; gastroenterites infecciosas e complicações; anemia; deficiências nutricionais; infecções de ouvido, nariz e garganta; pneumonias bacterianas; asma; doenças pulmonares; hipertensão; angina; insuficiência cardíaca; doenças cerebrovasculares; diabetes mellitus; epilepsia; infecção no rim e trato urinário; infecção da pele e tecido subcutâneo; doença inflamatória de órgãos pélvicos femininos; úlcera gastrointestinal e doenças relacionadas ao pré-natal e ao parto 4,5 .

Estudos têm documentado a relação inversa entre as medidas de acesso aos cuidados primários de saúde e as ICSAP 2,6. Por outro lado, o uso desse indicador vem sendo criticado por alguns pesquisadores que argumentam existirem outros elementos determinantes da hospitalização que estão fora do escopo de atuação da atenção primária à saúde, tais como as características inerentes ao paciente, fatores socioeconômicos e demográficos, a variabilidade da prática clínica hospitalar e as políticas de admissão nesses serviços 7,8,9.

Portanto, faz-se necessário o ajuste para essas variáveis confundidoras para se estudar a associação entre variáveis dos serviços de saúde e o indicador ICSAP. No Brasil, poucos estudos têm sido realizados com esse objetivo em um nível ecológico 10,11,12.

Nessa premissa, o presente estudo objetiva investigar a associação entre os determinantes socioeconômicos e as ICSAP. Além disso, será investigada a associação entre a disponibilidade de infraestrutura e cobertura de serviços de saúde com as ICSAP após o ajuste para variáveis socioeconômicas e demográficas no contexto dos municípios do Espírito Santo, Brasil.

\section{Métodos}

Foi realizado um estudo ecológico, com dados secundários das internações do Sistema Único de Saúde (SUS), para analisar as internações ocorridas nos 78 municípios do Estado do Espírito Santo de 1o de janeiro de 2010 a 31 de dezembro de 2010 .

As internações sensíveis foram agrupadas segundo idade e município de residência do paciente, para se evitar o viés criado ao ser utilizado o município de internação (maior número de internações nos municípios onde estão concentrados os hospitais).

Para o estudo descritivo, foram calculadas taxas de ICSAP expressas por 10 mil habitantes para cada município, na população total e por faixa etária, adotadas de acordo com os ciclos de vida (0-19 anos, 20-64 anos e 65 e mais). Enquanto, para o estudo analítico, o número de ICSAP constituíram a variável resposta, e a contagem da população por faixa etária foi incluída como offset do modelo.

A classificação como hospitalizações sensíveis à atenção primária seguiu a lista brasileira de ICSAP 5. Foi considerado como causa de internação o diagnóstico principal classificado conforme a 10a revisão da Classificação Estatística Internacional de Doenças e Problemas Relacionados à Saúde (CID-10). No total de internações, excluíram-se os partos por representarem um desfecho natural da gestação e por determinarem internações que ocorrem apenas na população feminina.

Os dados sobre as ICSAP foram obtidos dos arquivos de Autorização de Internação Hospitalar (AIH) pagas, registrados no Sistema de Informações Hospitalares (SIH) do SUS, disponibilizados na internet pelo Departamento de Informação e Informática do SUS (DATASUS).

A seleção de variáveis explicativas seguiu o modelo teórico proposto por Nedel et al. 13, no qual as taxas de hospitalização estão relacionadas a uma determinação hierárquica que, em seu nível distal, considera situação socioeconômica, estrutura social e características geográficas e demográficas de uma sociedade; no nível intermediário, encontram-se a influência da organização dos serviços de saúde (rede privada e pública), sua disponibilidade, barreiras 
de acesso e modelos de assistência à saúde oferecidos à população; e no nível proximal, estão os indicadores de desempenho do sistema de saúde. As variáveis explicativas elencadas neste estudo como proxy desses determinantes estão dispostas na Tabela 1.

Os municípios foram classificados segundo porte populacional, a escolha desse parâmetro para realizar a estratificação está em concordância com estudos que demonstraram haver diferenças na proporção das ICSAP, bem como no perfil de implantação da Estratégia Saúde da Família (ESF) e nos indicadores socioeconômicos conforme a população do município 10,14,15,16. Para o estudo descritivo, calcularam-se as medianas das variáveis para cada município com agrupamento por porte.

Inicialmente, calculou-se a correlação (Pearson) entre as variáveis para a eliminação das redundâncias geradas pela alta correlação que algumas variáveis poderiam apresentar entre si. Em seguida, foram realizadas a análise bivariada dos dados e, posteriormente, a multivariável para controlar pelas variáveis explicativas. Utilizou-se regressão de Poisson por se tratar de um modelo adequado para dados de contagem, tais como o número de internações aqui estudadas. Ademais, devido à confirmação de superdispersão, utilizaram-se estimadores robustos de variância.

As variáveis foram tratadas como contínuas nos modelos, e foram ajustados modelos para a população total e por faixas etárias.

A análise multivariável seguiu o modelo hierarquizado, efetivada em cinco etapas. $\mathrm{Na}$ primeira, realizou-se a regressão das variáveis explicativas do nível distal. Na segunda etapa, foram utilizadas as variáveis do nível distal que apresentaram valor de $\mathrm{p}<0,20$ após exclusão progressiva das que apresentavam os maiores valores de $\mathrm{p}$ (utilizou-se o método backward) e incorporaram-se as do nível intermediário.

Chegaram à terceira etapa as variáveis do nível distal e intermediário que apresentaram valor de $\mathrm{p}<0,20$ após exclusão progressiva das que apresentavam os maiores valores de $\mathrm{p}$ (utilizouse o método backward) e acrescentaram-se as do nível proximal. Na quarta etapa, encontram-se as variáveis do nível distal, intermediário e proximal que apresentaram valor de $\mathrm{p}<0,20$ após exclusão progressiva das que apresentavam os maiores valores de $\mathrm{p}$ (utilizou-se o método backward).

Na quinta e última etapa, chegou-se ao modelo final com todas as variáveis significativas (valor de $\mathrm{p}<0,05$ ). Também foram feitas análises de resíduos da regressão (q-q plot e análise de autocorrelação espacial por meio do índice de Moran) e de multicolinearidade (expresso pelo fator de expansão da variância) para o modelo final.
Para o processamento do banco de dados, foi usado o programa estatístico SPSS (SPSS Inc., Chicago, Estados Unidos), e, para as análises estatísticas, o software R versão 2.12.1 (The R Foundation for Statistical Computing, Viena, Áustria; http://www.r-project.org).

O projeto foi submetido ao Comitê de Ética em Pesquisa (CEP) do Hospital Infantil Nossa Senhora da Glória, da Secretaria de Saúde do Estado do Espírito Santo, que o liberou da necessidade de aprovação em CEP devido à utilização de informações de domínio público.

\section{Resultados}

Em 2010, ocorreram 198.148 internações no Espírito Santo, sendo 38.137 (22,41\%) ICSAP e 28 mil partos. Detectou-se um percentual pequeno, menor que $1 \%$, de perdas de informações das internações.

As maiores taxas de ICSAP ocorreram nos municípios de médio porte, com 159,4 por 10 mil habitantes, seguidos pelos de pequeno porte, 118,8 por 10 mil habitantes, grande porte I, 102,1 por 10 mil habitantes, e grande porte II, 55,5 por 10 mil habitantes (Tabela 2).

Quanto à idade, as taxas de ICSAP foram maiores entre os idosos dos municípios de médio porte, 698,0 por 10 mil habitantes, seguidos pela faixa etária pediátrica, 144,0 por 10 mil habitantes, e adultos, 114,1 por 10 mil habitantes. As menores taxas foram observadas nos municípios de grande porte II que chegaram a 194,9 por 10 mil habitantes entre idosos, seguidos pela faixa etária pediátrica, 68,9 por 10 mil habitantes, e adultos, 34,2 por 10 mil habitantes (Tabela 2).

Os municípios de grande porte II apresentaram os melhores resultados entre as variáveis socioeconômicas, seguidos pelos municípios de grande porte I. Observou-se a maior cobertura de ESF e do Programa de Agentes Comunitários de Saúde (PACS) entre os municípios de pequeno porte, enquanto a cobertura de plano de saúde foi maior entre os municípios de grande porte II, os quais também apresentaram o maior número de médicos por mil habitantes e o menor coeficiente de mortalidade infantil. A cobertura de pré-natal e o número de leitos hospitalares SUS foram maiores nos municípios de médio porte (Tabela 2).

Após a realização da correlação de Pearson entre todas as variáveis, foram excluídos o percentual de domicílios com abastecimento de água e o Índice de Desenvolvimento Humano Municipal (IDH-M), pois apresentaram alta correlação com taxa de urbanização e taxa de pobreza, respectivamente. 
Tabela 1

Modelo hierarquizado utilizado no estudo, variáveis explicativas e respectiva fonte.

\begin{tabular}{|c|c|c|}
\hline Variável * & Definições & Fonte \\
\hline \multicolumn{3}{|l|}{$\begin{array}{l}\text { Nível distal: socioeconômicas, geográficas e } \\
\text { demográficas }\end{array}$} \\
\hline Porte municipal (habitantes) & $\begin{array}{c}\text { Pequeno: } \leq \text { até 20.000; Médio: 20.001-100.000; Grande I:, 100.001-300.000; } \\
\text { Grande II: > 300.000 }\end{array}$ & IBGE \\
\hline Densidade demográfica & Razão entre o número de habitantes por área ocupada municipal & IBGE \\
\hline Taxa de urbanização & $\begin{array}{l}\text { Percentual da população residente em áreas urbanas } \\
\text { em relação à população total, em cada município }\end{array}$ & IBGE \\
\hline Taxa de analfabetismo & $\begin{array}{l}\text { Percentual das pessoas com } 15 \text { anos ou mais analfabetas } \\
\text { em relação ao total de pessoas do mesmo grupo etário }\end{array}$ & IBGE \\
\hline Proporção de pardos e negros & $\begin{array}{l}\text { Percentual de pessoas que se declararam pardas e pretas } \\
\text { em relação à população total }\end{array}$ & IBGE \\
\hline Densidade nos domicílios & $\begin{array}{l}\text { Percentual de pessoas que vivem em domicílios com densidade }>2 \text {, } \\
\text { razão entre o total de moradores do domicílio e o número total de cômodos } \\
\text { do domicílio, excluídos o(s) banheiro(s) e cozinha }\end{array}$ & PNUD \\
\hline Renda mensal familiar per capita & $\begin{array}{l}\text { Divisão do rendimento mensal dos componentes da família } \\
\text { pelo número de moradores da unidade domiciliar }\end{array}$ & IBGE \\
\hline Taxa de pobreza & $\begin{array}{l}\text { Percentual da população residente com renda familiar mensal per capita } \\
\text { de até meio salário mínimo }\end{array}$ & IBGE \\
\hline $\begin{array}{l}\text { Proporção de crianças em domicílio com } \\
\text { responsável/cônjuge não alfabetizado }\end{array}$ & $\begin{array}{l}\text { Percentual de crianças, } 0 \text { a } 5 \text { anos de idade, residentes em domicílios } \\
\text { com responsável ou cônjuge analfabeto }\end{array}$ & IBGE \\
\hline Domicílios com abastecimento de água & $\begin{array}{l}\text { Percentual de domicílios servidos por água canalizada } \\
\text { proveniente da rede geral de abastecimento }\end{array}$ & IBGE \\
\hline Domicílios com esgotamento sanitário & Percentual de domicílios com esgoto ligado à rede coletora ou à fossa séptica & IBGE \\
\hline PIB per capita & $\begin{array}{l}\text { Valor médio agregado por indivíduo dos bens e serviços finais produzidos pelos } \\
\text { municípios, em moeda corrente e a preços de mercado constantes de } 2008\end{array}$ & IJSN \\
\hline Índice de Gini & $\begin{array}{l}\text { Mede a desigualdade pela renda domiciliar per capita. } \\
\text { Se próximo de 1, maior é a desigualdade }\end{array}$ & IPEA \\
\hline IDH-M & $\begin{array}{l}\text { Considera a dimensão econômica, a longevidade e a educação. } \\
\text { Se próximo de } 1 \text {, maior é o desenvolvimento }\end{array}$ & PNUD \\
\hline IFDM & $\begin{array}{l}\text { Composto pela média dos IFDMs de emprego \& renda, educação e saúde. } \\
\text { Se próximo de } 1 \text {, maior é o desenvolvimento }\end{array}$ & FIRJAN \\
\hline \multicolumn{3}{|l|}{$\begin{array}{l}\text { Nível intermediário: organização dos serviços } \\
\text { de saúde }\end{array}$} \\
\hline Cobertura da ESF e do PACS & Percentual da população cadastrada pela ESF e pelo PACS & SIAB \\
\hline Cobertura de planos de saúde & $\begin{array}{l}\text { Percentual da população coberta por planos e } \\
\text { seguros de assistência suplementar à saúde }\end{array}$ & ANS \\
\hline Número de médicos & Número de médicos em atividade, por 1.000 habitantes & CNES \\
\hline Leitos hospitalares SUS & $\begin{array}{l}\text { Número de leitos hospitalares conveniados ou contratados pelo SUS, } \\
\text { por } 1.000 \text { habitantes residentes }\end{array}$ & AMS \\
\hline \multicolumn{3}{|l|}{$\begin{array}{l}\text { Nível proximal: indicadores de desempenho } \\
\text { dos serviços de saúde }\end{array}$} \\
\hline Coeficiente de mortalidade infantil & Óbitos de menores de um ano de idade, por 1.000 nascidos vivos & SIM/SINASC \\
\hline Cobertura de consultas de pré-natal & Proporção de nascidos vivos de mães com 7 e ou mais consultas de pré-natal & SINASC \\
\hline
\end{tabular}

AMS: Assistência Médico-Sanitária; ANS: Agência Nacional de Saúde Suplementar; CNES: Cadastro Nacional de Estabelecimentos de Saúde; ESF: Estratégia Saúde da Família; FIRJAN: Federação das Indústrias do Estado do Rio de Janeiro; IBGE: Instituto Brasileiro de Geografia e Estatística; IDH-M: Índice de Desenvolvimento Humano - Municipal; IFDM: Índice FIRJAN de Desenvolvimento Municipal; IJSN: Instituto Jones dos Santos Neves; IPEA: Instituto de Pesquisa Econômica Aplicada; PACS: Programa de Agentes Comunitários de Saúde; PIB: Produto Interno Bruto;

PNUD: Programa das Nações Unidas para o Desenvolvimento; SIAB: Sistema de Informação da Atenção Básica; SIM: Sistema de Informações sobre Mortalidade; SINASC: Sistema de Informações sobre Nascidos Vivos; SUS: Sistema Único de Saúde.

* Dados obtidos correspondem ao ano de 2010, com exceção do PIB per capita (2008), Índice de Gini (2003), IDH-M (2000), IFDM (2009) e Leitos hospitalares SUS por habitante (2009). 
Tabela 2

Distribuição das medianas, 1ํ e 3o quartil das taxas de internação por condições sensíveis à atenção primária e variáveis explicativas segundo porte municipal. Espírito Santo, Brasil, 2010.

\begin{tabular}{|c|c|c|c|c|}
\hline & \multicolumn{4}{|c|}{ Porte municipal } \\
\hline & Pequeno & Médio & Grande I & Grande II \\
\hline \multicolumn{5}{|l|}{ Variáveis do estudo } \\
\hline \multicolumn{5}{|l|}{ Taxas de ICSAP (por 10 mil habitantes) } \\
\hline População total & $118,8(75,0-233,5)$ & $159,4(116,1-246,5)$ & $102,1(88,9-113,5)$ & $55,5(49,7-59,6)$ \\
\hline \multicolumn{5}{|l|}{ Faixa etária (anos) } \\
\hline $0-19$ & $72,2(46,3-134,8)$ & $144,0(76,4-195)$ & $90,5(67,6-126,9)$ & $68,9(59,2-80,2)$ \\
\hline $20-64$ & $73,4(49,7-148,3)$ & $114,1(88,2-159,9)$ & $73,0(50,5-81,2)$ & $34,2(31,0-35,6)$ \\
\hline Acima de 65 & $584,6(332,2-908,5)$ & $698,0(530,2-897,9)$ & $523,9(324,7-532,7)$ & $194,9(171,4-210,3)$ \\
\hline \multicolumn{5}{|l|}{ Sexo } \\
\hline Masculino & $110,5(72,7-231,8)$ & $155,1(103,5-217,4)$ & $100,7(90,1-124,5)$ & $54,7(50,3-58,9)$ \\
\hline Feminino & $132,0(74,6-217,4)$ & $177,2(123,2-250,0)$ & $103,0(87,8-103,5)$ & $53,6(49,2-57,5)$ \\
\hline \multicolumn{5}{|l|}{ Variáveis explicativas } \\
\hline Densidade demográfica (habitantes $/ \mathrm{km}^{2}$ ) & $33,0(25,9-45,6)$ & $43,5(31,6-59,0)$ & $78,5(46,5-176,8)$ & $1.598,8(1.119,0-2.295,9)$ \\
\hline Taxa de urbanização (\%) & $52,3(38,0-68,2)$ & $67,0(60,4-77,9)$ & $88,0(86,0-91,4)$ & $99,4(98,7-99,6)$ \\
\hline Taxa de analfabetismo (\%) & $13,2(10,2-16,9)$ & $12,9(10,2-15,2)$ & $7,7(6,6-9,6)$ & $4,6(3,4-5,7)$ \\
\hline Proporção de pardos e negros (\%) & $48,4(40,5-56,5)$ & $53,5(44,6-65,9)$ & $57,7(52,9-63,2)$ & $61,0(54,5-66,8)$ \\
\hline Densidade nos domicílios (\%) & $10,4(7,9-12,5)$ & $13,0(10,0-15,0)$ & $17,5(15,2-18,8)$ & $16,7(13,7-19,6)$ \\
\hline Renda mensal familiar per capita (R\$) & $461,0(390,0-500,8)$ & $469,0(420,5-548,5)$ & $636,0(623,0-651,0)$ & $823,5(593,0-1.150,8)$ \\
\hline Taxa de pobreza (\%) & $33,7(29,4-40,9)$ & $35,3(28,5-38,5)$ & $23,3(21,0-25,0)$ & $17,4(13,1-21,7)$ \\
\hline $\begin{array}{l}\text { Proporção de crianças em domicílio } \\
\text { com responsável/cônjuge não } \\
\text { alfabetizado (\%) }\end{array}$ & $13,3(10,3-17,6)$ & $13,4(9,4-16,1)$ & $8,4(6,8-12,6)$ & $5,7(4,7-6,6)$ \\
\hline $\begin{array}{l}\text { Domicílios com abastecimento } \\
\text { de água (\%) }\end{array}$ & $58,3(43,2-72,4)$ & $69,9(57,6-80,3)$ & $88,7(85,8-89,8)$ & $97,9(97,1-98,8)$ \\
\hline $\begin{array}{l}\text { Domicílios com esgotamento } \\
\text { sanitário (\%) }\end{array}$ & \multicolumn{3}{|c|}{ sanitário (\%) } & $86,1(84,3-89,4)$ \\
\hline Produto Interno Bruto per capita (R\$) & $\begin{array}{c}8.628,8 \\
(7.447,3-10.512,8)\end{array}$ & $\begin{array}{c}9.504,0 \\
(8.557,0-14.372,0)\end{array}$ & $\begin{array}{c}11.920,0 \\
(9.410,8-15.485,1)\end{array}$ & $\begin{array}{c}21.552,2 \\
(13.590,2-36.819,3)\end{array}$ \\
\hline Índice de Gini (0 a 1) & $0,43(0,42-0,45)$ & $0,44(0,43-0,46)$ & $0,46(0,46-0,48)$ & $0,46(0,44-0,47)$ \\
\hline IDH-M (0 a 1) & $0,72(0,69-0,75)$ & $0,72(0,70-0,74)$ & $0,77(0,76-0,77)$ & $0,79(0,76-0,83)$ \\
\hline IFDM (0 a 1) & $0,67(0,65-0,69)$ & $0,69(0,65-0,73)$ & $0,74(0,73-0,77)$ & $0,8(0,79-0,82)$ \\
\hline Cobertura da ESF e do PACS (\%) & $99,9(98,3-102,2)$ & $95,2(88,3-100,1)$ & $68,4(59,9-79,4)$ & $36,8(24,1-51,4)$ \\
\hline Cobertura de planos de saúde (\%) & $8,3(4,7-12,6)$ & $9,9(7,3-15,9)$ & $23,7(22-26,1)$ & $46,0(39,1-57,1)$ \\
\hline Número de médicos (1.000 habitantes) & $0,6(0,4-0,9)$ & $0,7(0,5-1,1)$ & $1,6(0,9-2,3)$ & $2,0(1,8-2,8)$ \\
\hline Cobertura de planos de saúde (\%) & $8,3(4,7-12,6)$ & $9,9(7,3-15,9)$ & $23,7(22-26,1)$ & $46,0(39,1-57,1)$ \\
\hline Leitos hospitalares SUS (1.000 & $0,2(0,0-2,2)$ & $1,7(1,4-2,6)$ & $1,5(1,3-2,5)$ & $1,0(0,9-2,1)$ \\
\hline \multicolumn{5}{|l|}{ habitantes) } \\
\hline CMI (óbitos por 1.000 nascidos vivos) & $13,5(11,4-17,5)$ & $13,1(10,5-15,5)$ & $12,3(12,0-14,9)$ & $11,6(11,1-12,4)$ \\
\hline Cobertura de consultas de pré-natal (\%) & $62,5(54,7-74,4)$ & $70,2(56,0-75,3)$ & $55,4(53,5-80,0)$ & $64,8(62,1-69,0)$ \\
\hline
\end{tabular}

CMI: coeficiente de mortalidade infantil; ESF: Estratégia Saúde da Família; IDH-M: Índice de Desenvolvimento Humano - Municipal; IFDM: Índice FIRJAN de Desenvolvimento Municipal; PACS: Programa de Agentes Comunitários de Saúde; SUS: Sistema Único de Saúde. 
A análise bivariada entre as variáveis explicativas e o risco de ICSAP segundo população total e para cada extrato de faixa etária estão apresentados na Tabela 3. No que se refere ao porte municipal, nota-se tanto na população geral quanto no estudo por faixas etárias que o maior risco de ICSAP está nos municípios de médio porte, e há queda progressiva do risco nos municípios de pequeno porte, grande porte I e grande porte II.

A Tabela 3 também demonstra que a cobertura da ESF e do PACS apresentou discreta associação positiva com as ICSAP entre adultos, idosos e na população geral (RR = 1.01; IC95\%: 1.01-1.01).

As etapas intermediárias da análise hierarquizada (etapas de 1 a 4) e respectivo valores de significância (valor de p) na população total e por faixa etária são apresentadas na Tabela 4 .

No modelo final da análise hierarquizada, encontram-se as variáveis sem colinearidade e com significância estatística ( $\mathrm{p}<0,05)$ (Tabela 5).

A análise de resíduos da regressão por meio do gráfico de probabilidade normal (ou q-q plot) demonstra resíduos próximos da reta de 45o, indicando que os resíduos representam uma amostra aleatória (sem padrão). Quanto à verificação de dependência espacial dos resíduos, realizou-se análise de autocorrelação espacial pelo Índice de Moran. Como não há um teste de hipóteses apropriado para modelos log-lineares, como Poisson, transformou-se a variável resposta (CSAP) em logaritmo da taxa de CSAP, e foi calculado o Índice de Moran do modelo linear. Os resultados corroboram a hipótese nula de independência espacial dos resíduos.

Observou-se que, após ajuste para todas as variáveis, o percentual de urbanização, de analfabetismo e de leitos SUS ofertados mostrou-se positivamente associado ao risco de ICSAP na população geral, bem como nas faixas etárias (Tabela 5).

Associaram-se negativamente às internações sensíveis, a maior proporção de pardos e negros e cobertura de planos de saúde na população geral e nas faixas etárias. Na infância, somente a primeira foi significativa (Tabela 5).

Conforme observado na Tabela 5, na variável percentual de analfabetismo, foram detectados os maiores valores de associação com a ocorrência de ICSAP e alcançou o maior valor na faixa pediátrica $(\mathrm{RR}=1,17$; IC95\%: $1,13-1,21)$, seguida pela população geral $(\mathrm{RR}=1,11$; IC95\%: 1,07-1,16), adultos (RR = 1,10; IC95\%: 1,04-1,17) e idosos (RR = 1,08; IC95\%: 1,04-1,12).

A maior proporção de leitos SUS ofertados também esteve associada a maior risco de ICSAP e apresentou o maior valor na população geral (RR = 1,12; IC95\%: 1,05-1,19), seguida pela faixa etária pediátrica e adulta $(\mathrm{RR}=1,11$; IC95\%:
1,03-1,20) e idosos (RR = 1,09; IC95\%: 1,04-1,15) (Tabela 5).

\section{Discussão}

Este estudo permitiu uma reflexão geral sobre o uso do indicador ICSAP e não encontrou relação das ICSAP com a cobertura da ESF e do PACS. Por outro lado, na análise multivariável, observou-se que o percentual de urbanização, de analfabetismo e de leitos SUS ofertados esteve positivamente associado ao risco de internações sensíveis. Enquanto a cobertura de planos de saúde e a proporção de pardos e negros associaram-se negativamente às internações sensíveis.

No âmbito nacional, há relatos da diminuição das taxas de ICSAP associadas ao crescimento de cobertura da ESF 11,17. Ao contrário disso, no presente estudo, a cobertura da atenção primária à saúde, representada pela cobertura da ESF e do PACS, apresentou, na análise bivariada, uma discreta associação positiva com as internações sensíveis, ou seja, quanto maior a cobertura maior o número dessas hospitalizações.

Para explicar essa relação, pode-se pensar que, em regiões onde existia um estrangulamento no acesso aos serviços de saúde, há um aumento temporário nas hospitalizações ao ocorrer melhora no acesso, pois, nesse caso, estaria sendo atendida uma população sem assistência alguma anterior 4 .

De fato, no Espírito Santo, a atenção primária à saúde cresceu progressivamente na última década, a ESF, por exemplo, saltou de 13,2\% de cobertura, no ano 2000, para 58,8\% em 201018 . Porém, há que se considerar ainda a hipótese de falha nos processos de trabalho da atenção primária à saúde e, por isso, aumento das internações sensíveis.

Ao proceder ao ajuste das variáveis, observou-se que a cobertura da atenção primária à saúde não demonstrou relação com as ICSAP e foi excluída das etapas subsequentes por não ter alcançado valor estatisticamente significativo. Esse achado é importante, uma vez que o indicador se propõe a avaliar esse ponto da rede de atenção à saúde.

Diante disso, cabe questionar se os problemas na atuação da atenção primária à saúde podem ter culminado nesse resultado ou se a variável cobertura não é capaz de representar, por si só, o acesso à atenção primária à saúde e se faria necessário utilizar outra representação como, por exemplo, a utilização do serviço, a percepção dos usuários quanto à oportunidade de acesso e a adequação da implantação das equipes da ESF e do PACS, dentre outras 2,10. 
Análise bivariada entre variáveis explicativas e risco de internação por condições sensíveis à atenção primária na população total e por faixa etária. Espírito Santo, Brasil, 2010.

\begin{tabular}{|c|c|c|c|c|}
\hline \multirow[t]{3}{*}{ Variáveis } & \multirow{3}{*}{$\begin{array}{l}\text { População total } \\
\text { RR (IC95\%) }\end{array}$} & \multicolumn{3}{|c|}{ Faixa etária (anos) } \\
\hline & & $0-19$ & $20-64$ & Acima de 65 \\
\hline & & RR (IC95\%) & RR (IC95\%) & RR (IC95\%) \\
\hline \multicolumn{5}{|l|}{ Nível 1} \\
\hline Município porte grande II * & 1,00 & 1,00 & 1,00 & 1,00 \\
\hline Município porte grande I * & $2,17(2,11-2,24)$ & $1,29(1,22-1,36)$ & $2,79(2,66-2,93)$ & $2,54(2,38-2,71)$ \\
\hline Município porte médio * & $3,22(3,14-3,31)$ & $2,07(1,97-2,17)$ & $3,20(3,47-3,78)$ & $4,00(3,79-4,23)$ \\
\hline Município porte pequeno * & $3,01(2,92-3,11)$ & $1,74(1,64-1,85)$ & $3,46(3,30-3,64)$ & $3,48(3,28-3,7)$ \\
\hline Densidade demográfica & $1,00(0,99-1,00)$ & $1,00(0,99-1,00)$ & $1,00(0,99-1,00) * \star$ & $1,00(0,99-1,00)$ ** \\
\hline Taxa de urbanização & $0,99(0,99-0,99)$ & $0,99(0,99-0,99)$ & $0,99(0,98-0,99)$ & $0,98(0,98-0,99)$ \\
\hline Taxa de analfabetismo & $1,10(1,10-1,10)$ & $1,07(1,07-1,08)$ & $1,10(1,10-1,10)$ & $1,09(1,09-1,10)$ \\
\hline Proporção de pardos e negros & $0,98(0,98-0,98)$ & $0,99(0,99-0,99)$ & $0,99(0,98-0,99)$ & $0,98(0,98-0,98)$ \\
\hline Densidade nos domicílios & $0,93(0,93-0,94)$ & $0,95(0,94-0,95)$ & $0,94(0,94-0,94)$ & $0,94(0,94-0,95)$ \\
\hline Renda mensal familiar per capita & $1,00(0,99-1,00)$ & $1,00(0,99-1,00)$ & $1,00(0,99-1,00)$ & $1,00(0,99-1,00)$ \\
\hline Taxa de pobreza & $1,04(1,04-1,04)$ & $1,03(1,03-1,03)$ & $1,05(1,05-1,05)$ & $1,05(1,04-1,05)$ \\
\hline $\begin{array}{l}\text { Proporção de crianças em domicílio com responsável/ } \\
\text { cônjuge não alfabetizado }\end{array}$ & $1,09(1,09-1,09)$ & $1,06(1,06-1,06)$ & $1,10(1,09-1,10)$ & $1,09(1,09-1,10)$ \\
\hline Domicílios com esgotamento sanitário & $0,99(0,98-0,99)$ & $0,99(0,99-0,99)$ & $0,98(0,98-0,98)$ & $0,98(0,98-0,99)$ \\
\hline Produto interno bruto per capita & $1,00(1,10)$ & $1,00(1,10)$ & $1,00(1,10)$ & $1,00(1,10)$ \\
\hline Índice de Gini & $0,10(0,06-0,15)$ & $0,86(0,38-1,92)$ & $0,22(0,12-0,43)$ & $0,0(0,0)$ \\
\hline IFDM & $0,0(0,0)$ & $0,01(0,01-0,01)$ & $0,0(0,0)$ & $0,0(0,0) * \star$ \\
\hline \multicolumn{5}{|l|}{ Nível 2} \\
\hline Cobertura da ESF e do PACS & $1,01(1,01-1,01)$ & $1,01(1,00-1,01)$ & $1,01(1,01-1,01)$ & $1,01(1,01-1,02)$ \\
\hline Cobertura de planos de saúde & $0,97(0,97-0,98)$ & $0,99(0,99-0,99)$ & $0,97(0,97-0,97)$ & $0,97(0,97-0,97)$ \\
\hline Número de médicos & $0,74(0,74-0,75)$ & $0,90(0,89-0,92)$ & $0,69(0,68-0,70)$ & $0,70(0,68-0,71)$ \\
\hline Leitos hospitalares SUS & $1,06(1,05-1,06)$ & $1,10(1,09-1,11)$ & $1,03(1,02-1,04)$ & $1,01(1,00-1,02)$ \\
\hline \multicolumn{5}{|l|}{ Nível 3} \\
\hline $\mathrm{CMI}$ & $1,06(1,05-1,06)$ & $1,03(1,03-1,04)$ * & $1,07(1,07-1,08)$ & $1,05(1,04-1,05)$ * \\
\hline Cobertura de pré-natal & $1,00(0,99-1,00)$ & $0,99(0,99-0,99)$ & $1,00(1,00-1,00)$ & $1,00(0,99-1,00)$ * \\
\hline
\end{tabular}

CMI: coeficiente de mortalidade infantil; ESF: Estratégia Saúde da Família; IC95\%: intervalo de 95\% de confiança; IDH-M: Índice de Desenvolvimento Humano - Municipal; IFDM: Índice FIRJAN de Desenvolvimento Municipal; PACS: Programa de Agentes Comunitários de Saúde; RR: risco relativo; SUS: Sistema Único de Saúde.

* Correspondem às taxas de internação por condições sensíveis à atenção primária para cada grupo de porte municipal;

** $\mathrm{p}<0,01$

Nota: em negrito $p<0,001$, as demais variáveis não alcançaram $p<0,05$.

Pode-se tratar também de uma limitação do indicador ICSAP. Quanto a isso, Macinko 19 menciona que um dos entraves da sua utilização é o fato de que pode levar anos para que sejam estabelecidas tendências, assim como detecção de problemas em áreas pequenas e em mudanças pontuais. Outros estudos que demonstraram uma associação negativa da oferta de serviços de atenção básica e das internações sensíveis o fizeram por meio de análise de série temporal, enquanto, nesta pesquisa, considerou-se apenas o ano de 20102,11 .
Outro obstáculo do uso do indicador é a existência de características exógenas à atenção primária, isto é, atributos do paciente e do problema de saúde, entre os determinantes relevantes da internação 9,19. Nesse sentido, um diferencial do presente estudo foi considerar fatores influentes nas ICSAP para além da oferta da atenção básica como as características socioeconômicas, o perfil demográfico da população e os demais recursos de organização dos serviços de saúde.

A análise aqui apresentada mostra que a possível influência dos determinantes socioeconô- 
Tabela 4

Etapas intermediárias da análise hierarquizada das variáveis explicativas e risco de internação por condições sensíveis à atenção primária, valores de significância (valor de p), população total e faixa etária. Espírito Santo, Brasil, 2010.

\begin{tabular}{|c|c|c|c|c|c|c|c|c|c|c|c|c|c|c|c|c|}
\hline \multirow[t]{3}{*}{ Variáveis } & \multicolumn{4}{|c|}{ População total } & \multicolumn{12}{|c|}{ Faixa etária (anos) } \\
\hline & \multirow[b]{2}{*}{1} & \multirow[b]{2}{*}{2} & \multirow[b]{2}{*}{3} & \multirow[b]{2}{*}{4} & \multicolumn{4}{|c|}{$0-19$} & \multicolumn{4}{|c|}{$20-64$} & \multicolumn{4}{|c|}{ Acima de 65} \\
\hline & & & & & 1 & 2 & 3 & 4 & 1 & 2 & 3 & 4 & 1 & 2 & 3 & 4 \\
\hline \multicolumn{17}{|l|}{ Nível distal } \\
\hline Município porte grande I * & 0,16 & 0,30 & 0,14 & 0,15 & 0,32 & $\star \star$ & $\star *$ & 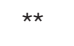 & 0,20 & 0,41 & 0,16 & 0,07 & 0,29 & 0,08 & 0,09 & 0,09 \\
\hline Município porte médio * & 0,14 & 0,24 & 0,19 & 0,20 & 0,15 & $\star \star$ & ** & $\star \star$ & 0,30 & 0,45 & 0,17 & 0,20 & 0,11 & 0,15 & 0,10 & 0,10 \\
\hline Município porte pequeno * & 0,17 & 0,28 & 0,23 & 0,22 & 0,39 & $\star \star$ & ** & $\star \star$ & 0,33 & 0,36 & 0,17 & 0,17 & 0,17 & 0,26 & 0,23 & 0,23 \\
\hline Densidade demográfica & 0,98 & $\star *$ & $\star \star$ & $\star \star$ & 0,35 & $\star \star$ & $\star \star$ & $\star \star$ & 0,73 & $\star \star$ & $\star \star$ & $\star \star$ & 0,41 & $\star \star$ & $\star \star$ & $\star \star$ \\
\hline Taxa de urbanização & 0,03 & 0,00 & 0,00 & 0,00 & 0,09 & 0,01 & 0,00 & 0,00 & 0,05 & 0,00 & 0,00 & 0,00 & 0,04 & 0,01 & 0,00 & 0,00 \\
\hline Proporção de pardos e negros & 0,01 & 0,00 & 0,00 & 0,00 & 0,02 & 0,00 & 0,00 & 0,00 & 0,13 & 0,01 & 0,00 & 0,00 & 0,00 & 0,01 & 0,00 & 0,00 \\
\hline Densidade nos domicílios & 0,65 & $\star \star$ & $\star \star$ & $\star \star$ & 0,90 & ** & ** & ** & 0,73 & $\star \star$ & $\star \star$ & $\star \star$ & 0,99 & ** & ** & $\star \star$ \\
\hline Renda mensal familiar per capita & 0,97 & $\star \star$ & $\star \star$ & $\star *$ & 0,44 & $\star \star$ & $\star \star$ & ** & 0,70 & $\star \star$ & $\star \star$ & $\star *$ & 0,87 & ** & ** & $\star \star$ \\
\hline Taxa de pobreza & 0,87 & $\star \star$ & $\star \star$ & $\star \star$ & 0,76 & ** & ** & ** & 0,62 & $\star \star$ & $\star \star$ & $\star \star$ & 0,97 & $\star \star$ & $\star \star$ & $\star \star$ \\
\hline Taxa de analfabetismo & 0,06 & 0,00 & 0,00 & 0,00 & 0,01 & 0,00 & 0,00 & 0,00 & 0,27 & 0,10 & 0,00 & 0,00 & 0,15 & 0,02 & 0,00 & 0,00 \\
\hline $\begin{array}{l}\text { Proporção de crianças em } \\
\text { domicílio com responsável/ } \\
\text { cônjuge não alfabetizado }\end{array}$ & 0,91 & $\star \star$ & $\star \star$ & $\star \star$ & 0,40 & $\star \star$ & $\star \star$ & $\star \star$ & 0,84 & $\star \star$ & $\star \star$ & $\star \star$ & 0,25 & $\star \star$ & $\star \star$ & $\star \star$ \\
\hline $\begin{array}{l}\text { Domicílios com esgotamento } \\
\text { sanitário }\end{array}$ & 0,28 & 0,56 & ** & $\star \star$ & 0,05 & 0,15 & 0,12 & 0,09 & 0,92 & ** & ** & ** & 0,16 & 0,23 & ** & $\star \star$ \\
\hline PIB per capita & 0,37 & $\star \star$ & $\star \star$ & $\star \star$ & 0,22 & 0,80 & & & 0,53 & $\star \star$ & $\star \star$ & $\star \star$ & 0,15 & 0,43 & $\star \star$ & $\star \star$ \\
\hline Índice de Gini & 0,48 & $\star \star$ & $\star \star$ & $\star \star$ & 0,45 & $\star \star$ & & & 0,57 & $\star \star$ & $\star \star$ & $\star \star$ & 0,91 & $\star \star$ & $\star \star$ & $\star \star$ \\
\hline IFDM & 0,13 & 0,18 & $\star \star$ & $\star \star$ & 0,11 & 0,13 & 0,00 & 0,00 & 0,13 & 0,14 & 0,25 & $\star \star$ & 0,51 & $\star \star$ & $\star \star$ & $\star \star$ \\
\hline \multicolumn{17}{|l|}{ Nível intermediário } \\
\hline Cobertura de planos de saúde & & 0,31 & 0,00 & 0,00 & & 0,47 & ** & ** & & 0,48 & 0,00 & 0,01 & & 0,12 & 0,00 & 0,00 \\
\hline Cobertura da ESF e do PACS & & 0,72 & ** & $\star \star$ & & 0,78 & $\star \star$ & $\star \star \star$ & & 0,36 & $\star \star$ & $\star \star$ & & 0,60 & ** & $\star \star$ \\
\hline Número de médicos & & 0,90 & $\star \star$ & $\star \star$ & & 0,70 & $\star \star$ & $\star \star$ & & 0,60 & $\star \star$ & $\star \star$ & & 0,90 & $\star \star$ & $\star \star$ \\
\hline Leitos hospitalares SUS & & 0,01 & 0,00 & 0,00 & & 0,01 & 0,00 & 0,00 & & 0,02 & 0,02 & 0,02 & & 0,05 & 0,01 & 0,01 \\
\hline \multicolumn{17}{|l|}{ Nível proximal } \\
\hline $\mathrm{CMI}$ & & & 0,64 & ** & & & 0,48 & ** & & & 0,22 & ** & & & 0,74 & $\star \star$ \\
\hline Cobertura de pré-natal & & & 0,12 & 0,12 & & & 0,19 & 0,17 & & & 0,07 & 0,07 & & & 0,20 & 0,18 \\
\hline
\end{tabular}

CMI: coeficiente de mortalidade infantil; ESF: Estratégia Saúde da Família; IFDM: Índice FIRJAN de Desenvolvimento Municipal; PACS: Programa de Agentes Comunitários de Saúde; SUS: Sistema Único de Saúde.

* Correspondem às taxas de internação por condições sensíveis à atenção primária para cada grupo de porte municipal;

** Excluídas da análise porque não alcançaram $p<0,20$ após exclusão progressiva das que apresentavam os maiores valores de $p$ (utilizou-se o método backward).

micos e demográficos nas ICSAP foi maior do que a cobertura da ESF e do PACS, pois eles permaneceram no modelo final tanto na população geral quanto nos estratos de faixa etária.

Sabe-se que piores condições socioeconômicas afetam o acesso aos serviços de saúde, modificam o padrão de utilização desses, dando preferência ao uso de serviços de emergência, prejudicam a adesão ao tratamento (falta de dinheiro para comprar medicamentos), dificultam a adoção de hábitos saudáveis e o amparo da rede de suporte social. Estudos prévios enfati- zaram a importância desses fatores e apontam para maiores taxas de ICSAP entre a população de piores condições socioeconômicas 7,8,20.

Neste estudo, encontrou-se o analfabetismo associado ao aumento das ICSAP, sendo seu maior efeito percebido na infância. Maiores taxas de analfabetismo denotam baixo padrão socioeconômico, e pode-se depreender daí que foi mais afetada, pelas ICSAP, a parcela da população com piores condições de vida ${ }^{21}$.

A baixa escolaridade leva a menor conhecimento em cuidados de saúde e interfere nas 
Modelo final da análise hierarquizada das variáveis explicativas e risco de internação por condições sensíveis à atenção primária na população total e por faixa etária. Espírito Santo, Brasil, 2010.

\begin{tabular}{|c|c|c|c|c|}
\hline \multirow[t]{3}{*}{ Variáveis } & \multirow{3}{*}{$\begin{array}{l}\text { População total } \\
\text { RR (IC95\%) }\end{array}$} & \multicolumn{3}{|c|}{ Faixa etária (anos) } \\
\hline & & $0-19$ & $20-64$ & Acima de 65 \\
\hline & & RR (IC95\%) & RR (IC95\%) & RR (IC95\%) \\
\hline \multicolumn{5}{|l|}{ Nível distal } \\
\hline Município porte grande II * & 1,00 & 1,00 & 1,00 & 1,00 \\
\hline Taxa de urbanização & $1,03(1,02-1,03)$ & $1,03(1,02-1,04)$ & $1,03(1,01-1,04)$ & $1,02(1,01-1,03)$ \\
\hline Proporção de pardos e negros & $0,97(0,96-0,98)$ & $0,97(0,96-0,98)$ & $0,98(0,96-0,99)$ & $0,98(0,97-0,99)$ \\
\hline Taxa de analfabetismo & $1,11(1,07-1,16)$ & $1,17(1,13-1,21)$ & $1,10(1,04-1,17)$ & $1,08(1,04-1,12)$ \\
\hline \multicolumn{5}{|l|}{ Nível intermediário } \\
\hline Cobertura de planos de saúde & $0,98(0,97-0,99)$ & $\star \star$ & $0,97(0,96-0,99)$ & $0,97(0,96-0,98)$ \\
\hline \multirow[t]{2}{*}{ Leitos hospitalares SUS } & $1,12(1,05-1,19)$ & $1,11(1,05-1,18)$ & $1,11(1,03-1,20)$ & $1,09(1,04-1,15)$ \\
\hline & & & $\star \star \star$ & \\
\hline Nível proximal & $\star \star$ & $\star \star$ & $\star \star$ & ** \\
\hline
\end{tabular}

IC95\%: intervalo de 95\% de confiança; RR: risco relativo; SUS: Sistema Único de Saúde.

Nota: o modelo final contempla as variáveis que, após a análise hierarquizada, alcançaram valor de $p<0,05$.

* Número de internação por condições sensíveis à atenção primária nos municípios porte grande II;

** Excluídas da análise porque não alcançaram $p<0,05$;

*** Essa variável alcançou $p<0,01$. As demais alcançaram $p<0,001$.

atitudes frente ao tratamento, pois dificulta o entendimento e pode prejudicar a adesão a ele. Além disso, a menor formação escolar diminui as chances de emprego, e os desempregados tendem a retardar a busca por cuidados de saúde. Essa associação entre baixa escolaridade e aumento das ICSAP também foi encontrada por outros autores 21,22 .

$\mathrm{O}$ achado, neste estudo, de maior número de ICSAP associadas à maior urbanização pode sugerir que a residência em aglomerados urbanos, nos quais, em geral, estão localizados os hospitais, facilita o acesso a eles, e, conforme aponta o estudo de Márquez-Calderón et al. ${ }^{8}$, o maior acesso aos serviços de urgência e de emergência hospitalares em detrimento da atenção básica colabora para o aumento das hospitalizações sensíveis, pois a atenção secundária e terciária passam a ser a porta de entrada do sistema de saúde.

A proporção de pardos e negros apareceu com uma relação negativa com as ICSAP, fato também observado em estudo realizado por Oliveira 12 . Conforme sugere ensaio publicado por Starfield et al. 23 , isso pode indicar que um sistema de saúde orientado para serviços de cuidados primários colabora na redução das disparidades de saúde entre grupos raciais, favorecendo a inserção no sistema de saúde dos menos favorecidos socialmente, como os negros.
De outra parte, estudos americanos revelam que, para categorias étnicas socialmente excluídas, é mais difícil o acesso aos serviços básicos de saúde, e, com isso, há carência de ações de prevenção, falta de diagnóstico e tratamento precoce, o que acarreta complicações das doenças e aumento das internações sensíveis 24,25.

Caberia, em estudos posteriores, averiguar se, de fato, a maior proporção de pardos e negros reduziu as internações sensíveis, porque essas populações estariam sendo mais assistidas por programas da atenção primária ou se o que está ocorrendo é um menor número de internações por restrição no acesso aos serviços de saúde por essa população.

Entre as variáveis que buscaram aferir a organização dos serviços de saúde, a cobertura de plano de saúde permaneceu no modelo final da análise hierarquizada realizada nesta pesquisa, na população geral, adultos e idosos. Notou-se que a sua maior ocorrência implicou menor número de ICSAP, acredita-se que a sua presença melhora o acesso a consultas preventivas de saúde e, por isso, reduza as internações sensíveis 1,12.

No entanto, deve-se considerar também que a população contratante dos serviços privados pode utilizar ou não os serviços públicos como também pode estar internada em hospitais privados, hospitalizações que ficaram fora do escopo deste estudo. A assistência privada atende a $25 \%$ da 
população brasileira, sendo que a proporção de contratantes é distribuída de forma bem distinta entre as cidades e chega a representar até $75 \%$ de cobertura em capitais como Vitória, Espírito Santo 26.

Desse modo, a implicação da cobertura de plano de saúde nas ICSAP reforça a sugestão feita por Alfradique et al. 4 de que, na análise das internações sensíveis, é necessário avaliar as taxas ajustadas também por cobertura de planos de saúde e regiões, pois, em áreas onde predominam o acesso à rede privada, poderá haver ampliação da obtenção de consultas preventivas de saúde, bem como menor utilização da rede hospitalar pública e, dessa forma, detecção de menores taxas de ICSAP, já que essas são mensuradas nos bancos do DATASUS.

Outro destaque do presente estudo foi a associação positiva entre a oferta de leitos hospitalares e as ICSAP, na população geral e nas faixas etárias pediátrica e idosa. Para alguns autores, isso se deve ao efeito de uma demanda induzida pela oferta, ou seja, quanto maior o número de leitos disponíveis, maior a chance de o indivíduo se internar independentemente das suas necessidades de saúde 15,27 .

Isso pode confirmar a afirmativa de que há fatores determinantes das internações sensíveis que fogem ao escopo de atuação da atenção primária à saúde, pois dizem respeito ao funcionamento da rede hospitalar, ao acesso à sua porta de entrada que são os serviços de urgência e de emergência, às práticas hospitalares de internação e aos critérios para se indicar uma internação ${ }^{3}$.
No que diz respeito ao porte dos municípios, em estudos prévios, foram encontradas menores taxas de ICSAP entre os municípios de maior porte, e, na medida em que se diminuía o tamanho dos municípios, ocorria aumento das ICSAP 10,15. No presente estudo, o porte municipal não esteve significativamente associado às internações sensíveis.

Por se tratar de um estudo ecológico, esta pesquisa não permite inferências individuais, mas houve o cuidado de se analisar o contexto municipal. Ademais, por estar baseada em dados secundários, encontra-se sujeita a limitações decorrentes de sub-registro nos bancos de dados, possíveis problemas de classificação como nos códigos de internação utilizados e eventual contagem dupla ou tripla de um mesmo paciente, pois o sistema não permite identificar reinternações.

Neste estudo, destacaram-se determinantes das internações que estão fora do campo de atuação da atenção primária à saúde e que também podem estar implicados no padrão de utilização dos serviços, tais como as características socioeconômicas, o perfil demográfico da população e a maior facilidade de acesso a serviços de saúde da atenção secundária e terciária, bem como da rede privada. Portanto, o estudo destaca a relevância desses fatores na ocorrência das internações sensíveis e sugere a necessidade de que eles sejam considerados quando se deseja avaliar as ICSAP como indicador de resultados da atenção primária. 


\section{Resumen}

El objetivo de este estudio fue investigar la asociación entre la organización de servicios de salud y las tasas de hospitalizaciones por ambulatory care sensitive conditions, ajustadas por variables socioeconómicas y demográficas, en el contexto de los municipios de Espírito Santo, Brasil. Estudio ecológico de ingresos del Sistema Único de Salud (SUS), con las variables: hospitalizaciones por ambulatory care sensitive conditions, tamaño municipal, determinantes demográficas, socioeconómicas y la organización de servicios de salud. El análisis multivariado se realizó mediante regresión de Poisson con ajuste robusto de la varianza. Se ordenaron variables explicativas por un modelo jerárquico. Se observó una asociación con el riesgo de las tasas de hospitalizaciones por ambulatory care sensitive conditions con analfabetismo (RR: 1,08-1,17), proporción de camas SUS (RR: 1,09-1,12), urbanización (RR: 1,021,03), proporción de negros (RR: 0,97-0,98) y cobertura del seguro de salud (RR: 0,97-0,98). Hay factores determinantes de hospitalizaciones por ambulatory care sensitive conditions que implican en el patrón de uso de los servicios de salud y se encuentran fuera del ámbito de la atención primaria.

Indicadores de Calidad de la Atención de Salud; Atención Primaria de Salud; Estudios Ecológicos

\section{Referências}

1. Laditka JN, Laditka BS, Probst JC. Health care access in rural areas: Evidence that hospitalization for ambulatory care-sensitive conditions in the United States may increase with the level of rurality. Health Place 2009; 15:761-70.

2. Ansari Z, Laditka JN, Laditka BS. Access to health care and hospitalization for ambulatory care sensitive conditions. Med Care Res Rev 2006; 63:719-41.

3. Caminal J. Las hospitalizaciones por ambulatory care sensitive conditions y los resultados de la atención primaria. Aten Primaria 2007; 39:525-34.

4. Alfradique ME, Bonolo PF, Dourado I, Lima-Costa MF, Macinko J, Mendonça CS, et al. Internações por condições sensíveis à atenção primária: a construção da lista brasileira como ferramenta para medir o desempenho do sistema de saúde (Projeto ICSAP - Brasil). Cad 2009; 25:1337-49.

5. Secretaria de Ações de Saúde, Ministério da Saúde. Portaria no 221, 17 de abril de 2008. Publica a lista brasileira de internações por condições sensíveis à atenção primária. Diário Oficial da União 2008; 18 abr.

\section{Colaboradores}

R. G. Pazó participou na concepção, projeto, análise, interpretação dos dados e redação do artigo. D. O. Frauches participou da concepção, projeto, análise dos dados e redação do artigo. M. C. B. Molina participou com revisão crítica relevante do conteúdo intelectual. N. V. Cade participou do projeto, redação do artigo e aprovação final da versão a ser publicada.
6. Macinko J, Oliveira VB, Turci MA, Guanais FC, Bonolo PF, Lima-Costa MF. The influence of primary care and hospital supply on ambulatory care-sensitive hospitalization among adults in Brazil, 1999-2007. Am J Public Health 2011; 101:1963-70.

7. Menéndez-Asenjo AA, Leal CF, Pena SS. Hospitalización evitable por ambulatory care sensitive conditions (ACSC) en la Comunidad de Madrid. Reflexiones sobre su uso como medida de resultado de la Atención Primaria. Rev Adm Sanit Siglo XXI 2003; 1:657-78.

8. Márquez-Calderón S, Rodríguez-Del Águilla MM, Perea-Milla E, Ortiz J, Bermúdez-Tamayo C. Factores asociados a la hospitalización por procesos sensibles a cuidados ambulatorios en los municipios. Gac Sanit 2003; 17:360-7.

9. Billings J, Zeitel L, Lukomnik J, Carey TS, Blank AE, Newman L. Impact of socioeconomic status on hospital use in New York City. Health Aff (Millwood) 1993; 12:162-73. 
10. Henrique F, Calvo MCM. Grau de implantação do Programa Saúde da Família e indicadores sociais. Ciênc Saúde Coletiva 2009; 14 Suppl 1:1359-65.

11. Mendonça CS, Harzheim E, Duncan BB, Nunes LN, Leyh W. Trends in hospitalizations for primary care sensitive conditions following the implementation of Family Health Teams in Belo Horizonte, Brazil. Health Policy Plan 2011; 27:348-55.

12. Oliveira AC. Ensaios sobre atenção pública à saúde em Minas Gerais [Dissertação de Mestrado]. Belo Horizonte: Centro de Planejamento Regional, Faculdade de Ciências Econômicas, Universidade Federal de Minas Gerais; 2007.

13. Nedel FB, Facchini LA, Bastos JL, Martin-Mateo M. Aspectos conceituais e metodológicos no estudo das hospitalizações por condições sensíveis à atenção primária. Ciênc Saúde Coletiva 2011; 16 Suppl 1:1145-54.

14. Instituto Brasileiro de Geografia e Estatística. Perfil dos municípios brasileiros: 2011. Rio de Janeiro: Instituto Brasileiro de Geografia e Estatística; 2012.

15. Souza LL, Dias-da-Costa JS. Internações por condições sensíveis à atenção primária nas coordenadorias de saúde no RS. Rev Saúde Pública 2011; 45:765-72.

16. Dias-da-Costa JS, Buttenbender DC, Hoefel AL, Souza LL. Hospitalizações por condições sensíveis à atenção primária nos municípios em gestão plena do sistema no Estado do Rio Grande do Sul, Brasil. Cad Saúde Pública 2010; 26:358-64.

17. Rehem TCMSB, Egry EY. Internações por condições sensíveis à atenção primária no Estado de São Paulo. Ciênc Saúde Coletiva 2011; 16:4755-66.

18. Departamento de Atenção Básica, Secretaria de Atenção à Saúde, Ministério da Saúde. Atenção básica e a saúde da família: números da saúde da família. http://dab.saude.gov.br/abnumeros.php (acessado em 10/Jan/2012).

19. Macinko J. III Seminário Internacional de Atenção Primária - Saúde da Família. Relatório de atividades. http://189.28.128.100/dab/docs/publicacoes/ geral/3seminario_internacional_relatorio_ativida des.pdf (acessado em 13/Jan/2012).
20. Roos LR, Walid R, Uhanova J, Bond R. Physician visits, hospitalizations, and socioeconomic status: ambulatory care sensitive conditions in a Canadian setting. Health Serv Res 2005; 40:1167-85.

21. Fernandes VBL, Caldeira AP, Faria AA, Rodrigues Neto JF. Internações sensíveis na atenção primária como indicador de avaliação da Estratégia Saúde da Família. Rev Saúde Pública 2009; 43:928-36.

22. Nedel FB, Facchini LA, Martín-Mateo M, Vieira LAS, Thumé E. Programa Saúde da Família e condições sensíveis à atenção primária, Bagé (RS). Rev Saúde Pública 2008; 42:1041-52.

23. Starfield B, Shi L, Macinko J. Contribution of primary care to health systems and health. Milbank Q 2005; 83:457-502.

24. Biello KB, Rawlings J, Carroll-Scott A, Browne R, Ickovics JR. Racial disparities in age at preventable hospitalization among U.S. adults. Am J Prev Med 2010; 38:54-60.

25. Laditka JN, Laditka BS, Probst JC. More may be better: evidence of a negative relationship between physician supply and hospitalization for ambulatory care sensitive conditions. Health Serv Res 2005; 40:1148-66.

26. Agência Nacional de Saúde Suplementar. Caderno de Informação da Saúde Suplementar: beneficiários, operadoras e planos. http://www.ans.gov.br/ anstabnet/anstabnet/materia_novo.htm (acessado em 10/Mai/2012).

27. Castro MSM, Travassos C, Carvalho MS. Efeito da oferta de serviços de saúde no uso de internações hospitalares no Brasil. Rev Saúde Pública 2005; 39:277-84.

Recebido em 15/Mai/2013

Versão final reapresentada em 16/Fev/2014

Aprovado em 19/Fev/2014 